\title{
Refining complex models with FWI using long-offset towed-streamer acquisitions
}

\section{N. Chemingui* and A.A. Valenciano, PGS}

Copyright 2019, SBGf - Sociedade Brasileira de Geofísica

This paper was prepared for presentation during the $16^{\text {th }}$ International Congress of the Brazilian Geophysical Society held in Rio de Janeiro, Brazil, 19-22 August 2019.

Contents of this paper were reviewed by the Technical Committee of the $16^{\text {th }}$ International Congress of the Brazilian Geophysical Society and do not necessarily represent any position of the SBGf, its officers or members. Electronic reproduction or storage of any part of this paper for commercial purposes without the written consent of the Brazilian Geophysical Society is prohibited.

\begin{abstract}
Velocity model building (VMB), in the presence of complex salt bodies, is an interpretive process that can produce suboptimal results. We discuss the application of Full Waveform Inversion (FWI) to automate the refinement of legacy velocity models generated by standard VMB workflows. We validate our solution using long-offset synthetic and field data examples from the Gulf of Mexico. The long offsets and low frequencies were key to using both refractions and reflections to update the deeper parts of the velocity model. We use an FWI velocity gradient that eliminates the migration isochrones; thereby removing the reflectivity imprint from the model updates. The application of FWI to the legacy models successfully refined the geometry of the salt bodies, including the base salt and the intra-salt enclosures. It also improved the RTM images; particularly the salt flanks and the subsalt reflectors.
\end{abstract}

\section{Introduction}

Full Waveform Inversion (FWI) is the tool of choice for building high-resolution velocity models. It involves nonlinear minimization of the misfit between the recorded and modelled seismic data while iteratively updating the subsurface model. The success of FWI depends on the seamless recovery of the short- and long-wavelength features missing in the starting velocity model.

Most FWI applications have targeted shallow water environments where the recorded refracted and diving waves enable the inversion to resolve the small-scale geologic features up to the deepest turning point (e.g. Sirgue et al., 2009; Zhou et al., 2015). Recently, there have been successful FWI applications in deep-water scenarios where refracted and diving waves are often missing due to limited far offsets in towed-streamer acquisitions. Consequently, there has been a growing demand for acquiring better data for FWI, e.g., longer offsets from ocean bottom seismic (Shen et al., 2017) and lower frequencies with a high signal-to-noise ratio (Dellinger et al., 2016). Alternatively, FWI developments have focused on better inversion solutions that can reduce the data requirements and produce deep model updates. These efforts have targeted combinations of modified gradients, robust norms for measuring the data misfit, and a priori model constraints to enable utilization of all wave modes in the data (reflections, refractions, and diving waves). They can reduce the data requirements at the expenses of more sophisticated workflows.
We combine acquisition strategies that enable long offset data with a robust FWI solution that utilizes both reflections and transmitted arrivals to invert for highresolution velocity models in complex regimes.

\section{The FWI algorithm}

Our inversion algorithm uses time-domain wave propagation and a normalized form of the Born scattering kernel to compute the FWI gradient (Tarantola, 1984). We solve the two-way anisotropic wave equation using the pseudo analytic (PA) method (Ramos-Martinez et al., 2011). We use a variable-density implementation for better matching of the relative amplitudes; particularly from the water-bottom reflections and high-contrast interfaces. We implemented a robust velocity gradient derived from Inverse Scattering theory and impedancevelocity parameterization of FWI (Ramos-Martinez et al., 2016). This eliminates the migration isochrones that dominate conventional cross-correlation FWI gradients (Figure 1).

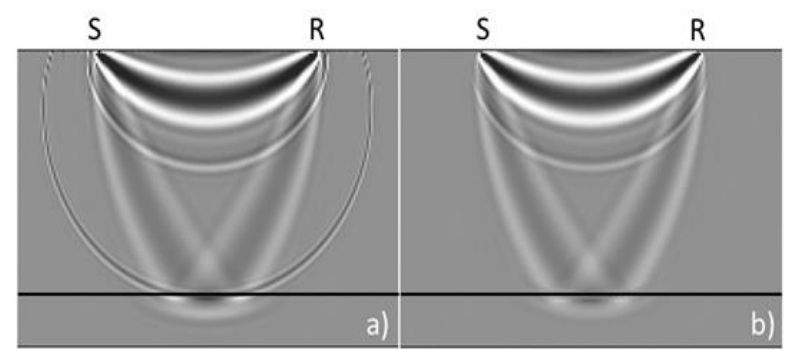

Figure 1. Sensitivity kernels for a source-receiver pair in a model with a $V(z)$ layer over a half-space. (a) Conventional cross-correlation FWI gradient; and (b) FWI velocity gradient for long wavelength model updates. The implementation of (b) eliminates the migration isochrones and removes the reflectivity footprint

FWI with long-offset data: a synthetic example

We generated a synthetic dataset to address the realistic imaging and model building challenges associated with complex salt structures. The model was inspired from an existing Gulf of Mexico deep-water scenario. The workflows, however, are applicable to other salt provinces (e.g. West Africa, Brazil, etc...). The synthetic data included offsets up to $40 \mathrm{~km}$ and low frequencies that can realistically be recorded in streamer surveys using air gun source arrays (Figure 2).

The starting model for FWI was a biased and heavily smoothed version of the true model. The input data for the inversion were selected to minimize the contribution from reflections so that $\mathrm{FWI}$ would mainly utilize the transmission modes. The inversion was carried out to a maximum frequency of $4 \mathrm{~Hz}$.

At a first stage the offset was limited to $15 \mathrm{~km}$ and the updates are shown in Figure 3b. FWI was able to improve 
the shallow sediment model, the top salt, and the shallow salt flanks. After increasing the offset to $30 \mathrm{~km}$, the inversion produced deeper updates that better matched the true model up to the base salt (Figure 3c).

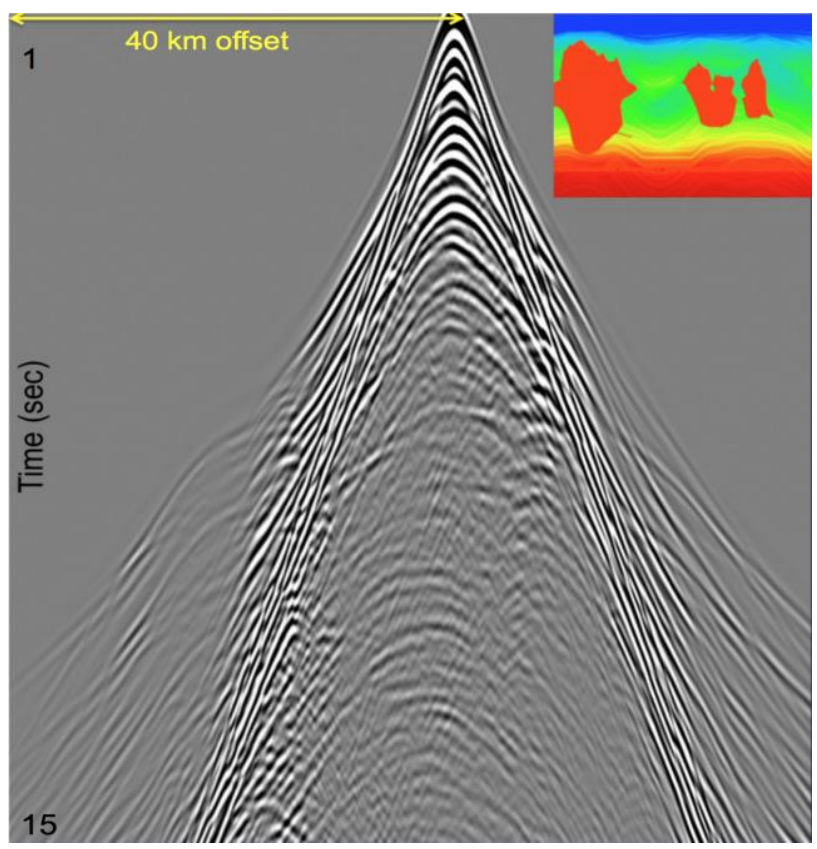

Figure 2. Synthetic shot record and velocity model (right top corner). The data are rich in long-offset transmitted events as well as reflected arrivals.

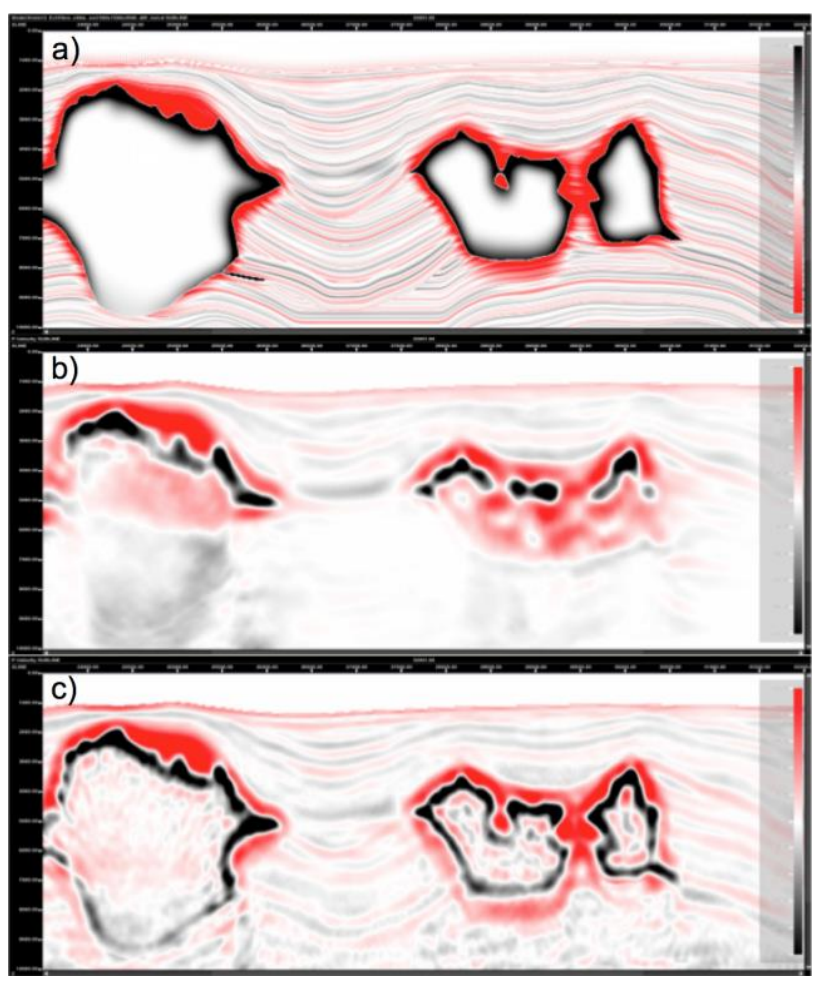

Figure 3. a) Difference between true and starting models, b) FWI updates using $15 \mathrm{~km}$ maximum offset data, and c) FWI updates using $30 \mathrm{~km}$ offsets.

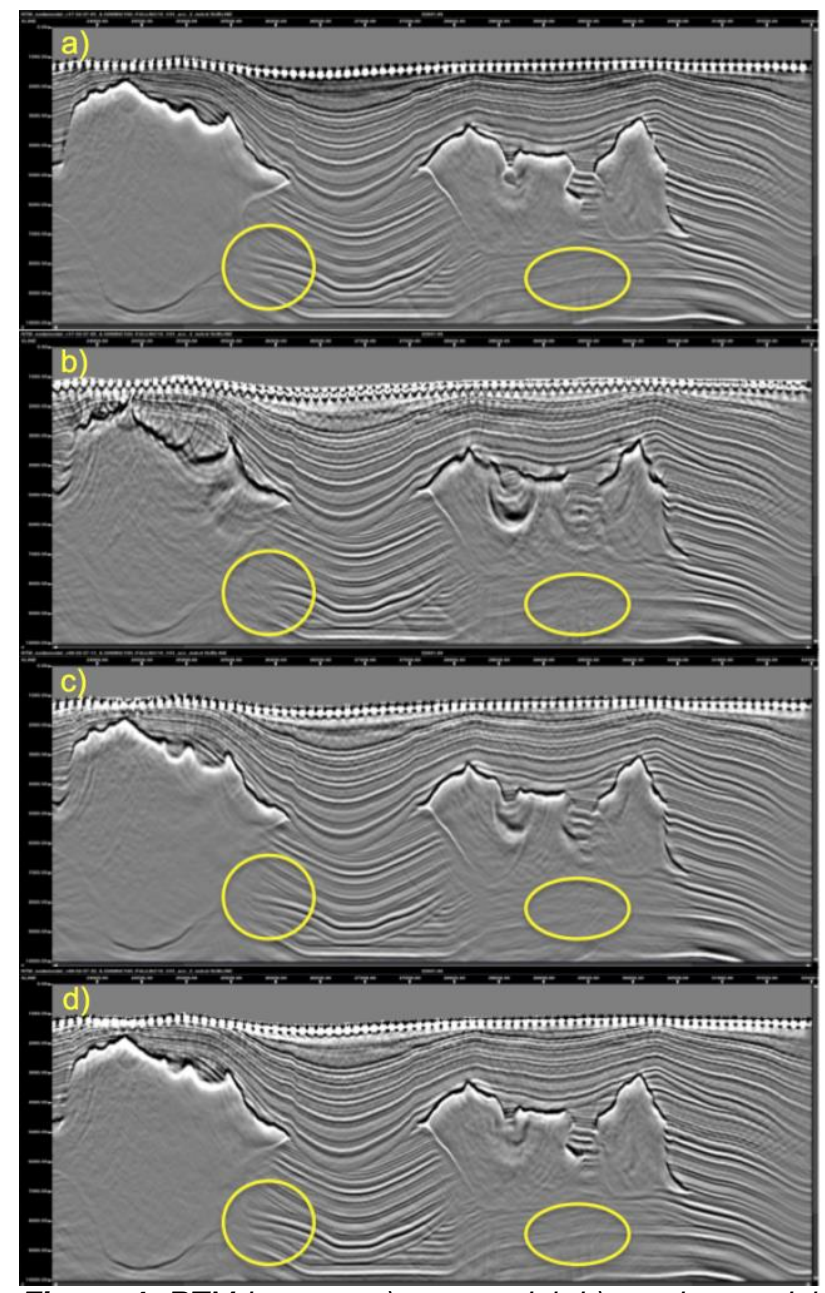

Figure 4. RTM images: a) true model, b) starting model, c) FWI with $15 \mathrm{~km}$ maximum offset, and d) FWI with $30 \mathrm{~km}$ maximum offset.

The updates were further validated by the RTM images (Figure 4). Migrating with the initial model resulted in a poor image particularly for the salt flanks, top salt, and sediment truncations. Using the $15 \mathrm{~km}$ FWI model we were able to resolve most of the shallow part (up to $6 \mathrm{~km}$ depth). By including the ultra-long offsets (up to $30 \mathrm{~km}$ ), we successfully recovered a clear image of the base salt and the deep subsalt sediments up to $10 \mathrm{~km}$ depth.

\section{Application to field data}

The field survey was designed to acquire long-offset, fullazimuth (FAZ) data in the Central and Western planning areas in the Gulf of Mexico (Long et al., 2014). Two streamer vessels were used each towing ten, $8-\mathrm{km}$ dualsensor streamers in combination with three additional source vessels in a SLO configuration.

To minimize the likelihood of cycle skipping, we performed a multi-stage FWI starting with data that exhibited coherent signal in the $2-4 \mathrm{~Hz}$ frequency band. The initial velocity model was generated using an interpretive VMB workflow including wavelet-shift 
tomography and salt interpretation following a top-down strategy.

Figure 5 shows a comparison of the updates using only refractions (left) and a combination of refractions and reflections (right). Note that the refracted modes produce reliable velocity updates up to $6 \mathrm{~km}$ depth. In contrast, our robust FWI velocity gradient is able to use the reflections to update the velocity model beyond the penetration depth of diving waves. Figure 6 shows a comparison of the RTM images from the initial (left) and the FWI (right) velocity models. The FWI velocity model improves the image of the salt boundaries (top, bottom, and flanks) as well as the sediment truncations against the salt. Similarly, the deeper reflectors display improved continuity after FWI. Figure 7 illustrates the process of refining the velocity model by FWI. The errors in the salt interpretation (bottom left) are corrected by FWI that removes salt (blue updates) and adds salt (red updates), as needed.

\section{Conclusions}

We discussed an automated workflow for refining velocity models in complex regimes by using data from long-offset recordings and by employing a robust FWI gradient for deep model updating. The synthetic model demonstrated that transmission-only FWI requires ultra-long offsets to resolve the deeper parts of the velocity model. In contrast, our robust FWI velocity gradient successfully utilized the reflections in the field survey to update the velocity model beyond the penetration depth of diving waves. Using all wave modes, FWI was able to refine the sediment velocities and repair the geometry of the salt including the intra-salt enclosures. It also improved the RTM images particularly the salt flanks and the subsalt reflectors.

\section{Acknowledgments}

We thank PGS MultiClient for permission to use the field data. We also thank Jan Egil Kirkebø for assistance with the field data, and Lingyun Qiu, Dan Whitmore, and Faqi Liu for helpful discussions.

\section{References}

Dellinger, J., Ross, A., Meaux, D., Brenders, A., Gesoff, G., Etgen, J., Naranjo, J., Openshaw, G., and Harper, M., 2016, Wolfspar, an 'FWI-friendly' ultralow-frequency marine seismic source: 86th Meeting, SEG, Dallas, Expanded Abstracts, 4891-4895.
Long, A., Campbell, S., Fishburn, S., Brandsberg-Dahl, S., Chemingui, N., and Dirks, V., 2014, No-compromise marine seismic: A full-azimuth survey design for totalwavefield velocity model building and imaging in the deep-water Gulf of Mexico: 84th Meeting, SEG, Denver, Expanded Abstracts, 52-56.

Ramos-Martinez, J., Crawley, S., Kelly, S., and Tsimelzon, B., 2011, Full-waveform inversion by pseudoanalytic extrapolation: 81st Meeting, SEG, San Antonio, Expanded Abstracts, 2684-2688.

Ramos-Martinez, J., Crawley, S., Zou, Z., Valenciano, A.A., Qiu, L. and Chemingui, N., 2016, A robust gradient for long wavelength FWI updates: 76th Meeting, EAGE, Vienna, Extended Abstracts, Th SRS2 03.

Shen, X., Ahmed, I., Brenders, A., Dellinger, J., Etgen, J., and Michell, S., 2017, Salt model building with fullwaveform inversion: 87th Meeting, SEG, Anaheim, Expanded abstracts, 1507-1511.

Sirgue, L., Barkved, O.I., Van Gestel, J.P., Askim, O.J. and Kommedal, J.H., 2009, 3D waveform inversion on Valhall wide-azimuth OBC: 71st Meeting, EAGE, Amsterdam, Extended Abstracts, U038.

Tarantola, A., 1984, Inversion of seismic reflection data in the acoustic approximation: Geophysics, 49, 1259-1266.

Zhou, W., Brossier, R., Operto S., and Vireux, J., 2015, Full waveform inversion of diving waves for velocity model building with impedance inversion based on scale separation: Geophysical Journal International, 202, 15351554. 

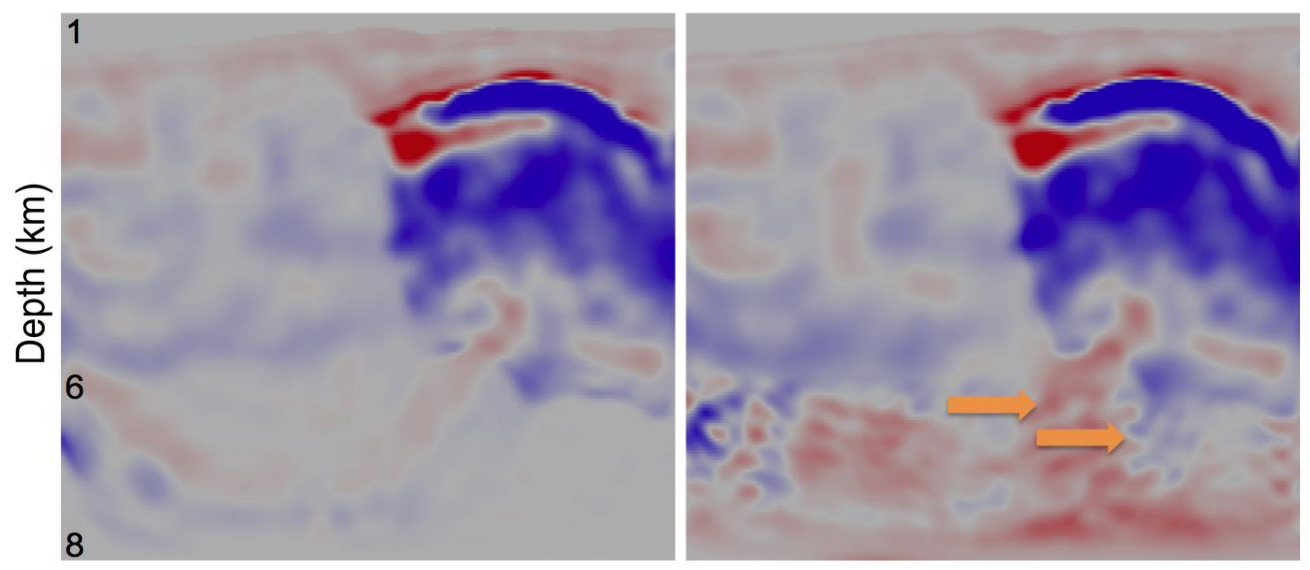

Figure 5: FWI updates comparison: using only refractions (left), vs. using combined refractions and reflections (right). Reflections can update the long wavelength components of the velocity model beyond the penetration depth of diving waves (orange arrows).

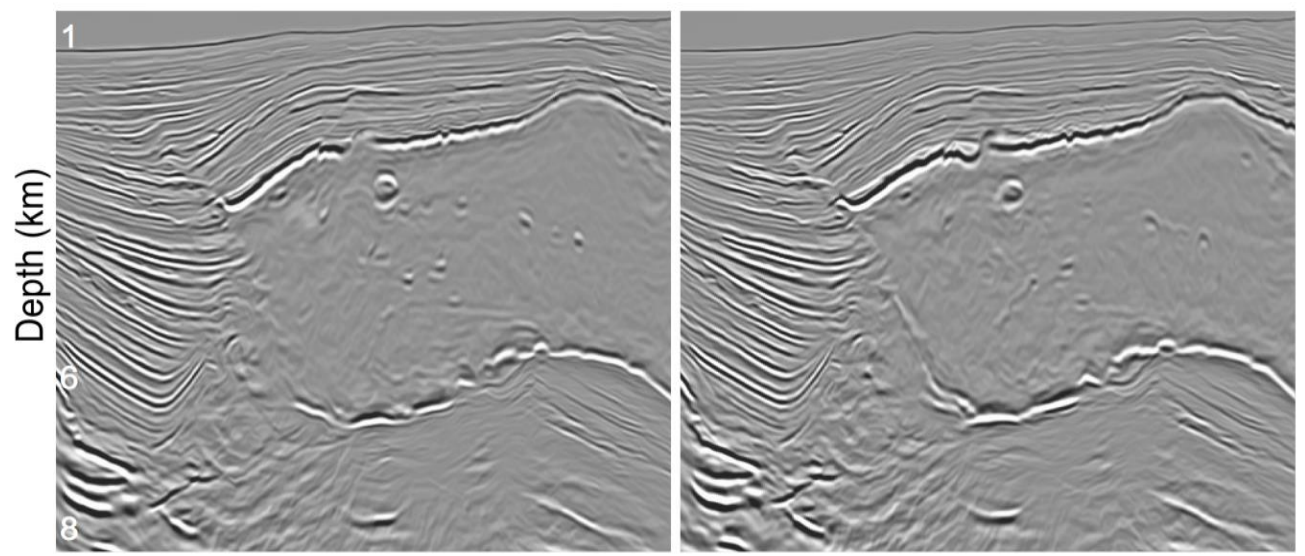

Figure 6: RTM images comparison: initial (left), vs. FWI (right). Note how the FWI model improves the imaging of the salt boundaries and the sediment truncations.
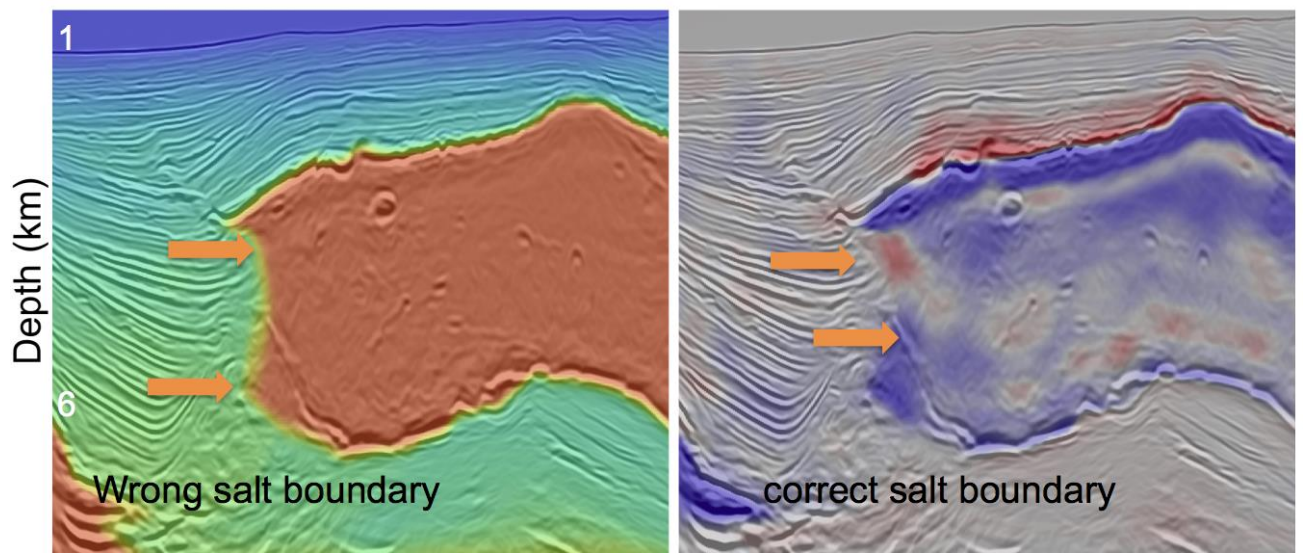

Figure 7: Legacy velocity overlaid on the FWI RTM image (left), and FWI model updates overlaid on the FWI RTM image (right). Note how FWI corrects the salt interpretation by "removing" salt (blue update) and "adding" salt (red update) as needed (areas marked by orange arrows). 